\title{
Effect of body temperature on visual evoked potential delay and visual perception in multiple sclerosis
}

\author{
D. RE G A N, T. J . MURRA Y, A N D R. S I L V E R \\ From the Department of Psychology and Department of Medicine (Camp Hill Hospital), \\ Dalhousie University, Halifax, Nova Scotia, Canada
}

S UM MAR Y Seven multiple sclerosis patients were cooled and four heated, but evoked potential delay changed in only five out of 11 experiments. Control limits were set by cooling eight and heating four control subjects. One patient gave anomalous results in that although heating degraded perceptual delay and visual acuity, and depressed the sine wave grating MTF, double-flash resolution was improved. An explanation is proposed in terms of the pattern of axonal demyelination. The medium frequency flicker evoked potential test seems to be a less reliable means of monitoring the progress of demyelination in multiple sclerosis patients than is double-flash campimetry or perceptual delay campimetry, although in some situations the objectivity of the evoked potential test would be advantageous.

Neurophysiological studies of single neurones have shown that raising the temperature of normal or partially demyelinated axons slightly increases conduction velocity until just below the temperature at which axonal conduction fails (the blocking temperature). Raising the temperature still further produces a marked fall of conduction velocity. Demyelination can so reduce the blocking temperature that conduction fails at temperatures only slightly above normal body temperature (Davis, 1970; Raminsky, 1973; Schauf and Davis, 1974).

Such neurophysiological observations on single axons go some way to explaining why exercise or heating may worsen signs and symptoms in some multiple sclerosis patients, and may even cause new signs and symptoms to appear (Uhthoff, 1890; Davis, 1966; Namerow, 1971). These single neurone data can also account for the temporary improvement that may accompany cooling (Watson, 1959; Boynton et al., 1959; Symington et al., 1977), and can explain why very small changes in body temperature-for example, $0.1^{\circ} \mathrm{F}$-or even daily circadian variations (Namerow, 1968; Davis et al., 1973) can be sufficient to produce large effects in patients with multiple sclerosis. In the well known 'hot bath' provocation test the effects of heating are usefully used to help the diagnosis of multiple sclerosis (Davis, 1966).

Address for reprint requests : Dr D. Regan, Department of Psychology Dalhousie University, Halifax, Nova Scotia, Canada B3H 4JL. Accepted 25 June 1977
A quite separate point is that temperature manipulations might provide a means of assessing the effectiveness of techniques for monitoring the progress of multiple sclerosis on the grounds that, in some patients, small variations of body temperature that are well within physiological limits can mimic the symptomatic effect both of increasing demyelination and of remyelination. We have previously reported on double-flash campimetry as a possible means of monitoring the progress of demyelination and of detecting possible remyelination (Galvin et al., 1976b). Evoked potential recording has recently proved to be a diagnostically useful indication of visual pathology in multiple sclerosis, and this method has the advantage of objectivity (Regan, 1977a; Halliday and McDonald, 1977; McDonald and Halliday, 1977). Here we report on evoked potential recording as a possible objective means of monitoring the progress of demyelination in multiple sclerosis.

\section{Methods}

EVOKED POTENTIAL RECORDING

Subjects viewed a diffusing panel subtending $48^{\circ} \times 48^{\circ}$ from a distance of $330 \mathrm{~mm}$. The panel was lit uniformly by white light to a luminance of $110 \mathrm{~cd} / \mathrm{m}^{2}$. The panel's luminance could be controlled electronically and was sine wave flickered about the mean value $(30 \%$ modulation depth in most experiments) by feeding the appropriate electrical waveform to the driving electronics. One 
of the subject's eyes was occluded by an eyepatch.

Two methods were used to record 'medium frequency' flicker evoked potentials (EP). The 'single stimulation method' was to flicker the stimulus light with a sine wave signal of frequency $\mathrm{F} \mathrm{Hz}$. The steady state EP of frequency $\mathrm{F} \mathrm{Hz}$ was recorded by means of a Fourier analyser and its amplitude and phase recorded as described in Regan (1966) and Milner et al. (1974). Flicker frequency $F$ was varied between about $13 \mathrm{~Hz}$ and $28 \mathrm{~Hz}$. A plot of EP phase versus frequency gave the EP's 'apparent latency' (Regan, 1966; Milner et al., 1974). A speedier method was used in some experiments. Three sine waves of different frequencies $(\mathrm{F} 1, \mathrm{~F} 2$, and $\mathrm{F} 3 \mathrm{~Hz})$ were added together and this waveform flickered the stimulator. Three Fourier analysers, locked to frequencies F1, F2, and $\mathrm{F} 3 \mathrm{~Hz}$ respectively, simultaneously extracted three EPs from a single electrode derivation and displayed the amplitudes and phases of the three EPs in polar coordinates (Regan, 1976). This 'simultaneous stimulation method' directly sampled the slope of the phase versus frequency plot and thus measured EP latency with greater precision than the single stimulation method, since estimates were less affected by EP variability and nonstationarity (Regan, 1976).

\section{MEASUREMENT OF PERCEPTUAL DELAY}

Perceptual delay was measured using the procedure described previously (Heron et al., 1974; Regan et al., 1976a). The left eye viewed a small $\left(0.3^{\circ}\right.$ diameter solid-state red lamp and the right eye viewed a similar lamp. One lamp was placed slightly above the other, and both fell on the central fovea. One lamp was switched on a little later $(\mathrm{t} \mathrm{ms})$ than the other, and the value of $t$ was adjusted so that the lamps appeared to switch on simultaneously. This value $t \mathrm{~ms}$ then gave perceptual delay for one fovea relative to the other fovea.

\section{MEASUREMENT OF DOUBLE-FLASH RESOLUTION}

Double-flash resolution was measured using the procedure described previously (Regan, 1972b; Galvin et al., 1976a, b). Subjects viewed a single small red lamp $\left(0.3^{\circ}\right.$ diameter $)$ from a distance of one metre. The background was a diffuse white surface illuminated to a luminance of roughly $\mathbf{5 0}$ $\mathrm{cd} / \mathrm{m}^{2}$. The lamp delivered a pair of brief $(10 \mathrm{~ms})$ flashes separated by a preset interval. Using the method of ascending and descending limits the least interval between the flashes was found for which the two flashes could be seen as double. The fovea of each eye was tested separately. The unused eye was occluded during tests.
MEASUREMENT OF MODULATION TRANSFER FUNCTION USING A SINE WAVE GRATING STIMULUS

Standard tests for visual acuity-for example, the Snellen test-assess visual sensitivity for fine detail only. Visual sensitivity for coarse and medium detail as well as fine detail can be tested by using a sine wave grating stimulus whose spatial contrast is varied (a sine wave grating looks like a blurred grating of bright and dim bars). Our method was conventional and has been described elsewhere (Regan et al., 1977a). Sine wave grating stimuli were displayed on a cathode ray oscilloscope by electronic means. Spatial frequency was defined as the number of (bright plus dim) bars per degree of visual angle, and this could be varied. Contrast was defined as $I_{\max }-I_{\min }$, where $\overline{I_{\max }+I_{\min }}$

$I_{\max }$ and $I_{\min }$ were the luminances of a bright and dim bar respectively. For each given spatial frequency the contrast was reduced so as to find the contrast for which the grating could just be seen. Thus, the contrast threshold was a measure of visual sensitivity for each of the spatial frequencies tested. The stimulus field subtended $3.5^{\circ} \times 4.5^{\circ}$ (horizontally), was of mean luminance $17 \mathrm{~cd} / \mathrm{m}^{2}$, and had a white surround of luminance $11 \mathrm{~cd} / \mathrm{m}^{2}$ subtending about $21^{\circ} \times 14^{\circ}$ (horizontally).

\section{COOLING PROCEDURE}

Subjects ingested $400 \mathrm{ml}$ of finely crushed ice, within five to 10 minutes, either unflavoured or flavoured with orange juice, as in Galvin et al. (1976b).

\section{WARMING PROCEDURE}

Both legs were immersed to upper shin level in warm water at about $44^{\circ} \mathrm{C}$, and the subject was wrapped in blankets with two hot water bottles around the abdomen. Warming was continued until the patient became almost intolerably hot or until the patient reported markedly degraded vision: this took on average roughly $30 \mathrm{~min}$.

\section{Control subjects and patients}

Control subjects were matched for age to individual patients. The diagnostic criteria for multiple sclerosis were those set out by Schumacher et al. (1968). Patients were classified into clinically definite, probable, and possible groups according to the scheme described by Rose et al. (1976). In addition, each patient was rated on the 10 point disability scale of Kurtzke (1965). A total of 10 multiple sclerosis patients were tested in this EP study. Clinical details are summarised in the Table. 
Table Clinical summary and results for the 10 patients tested by evoked potential recording

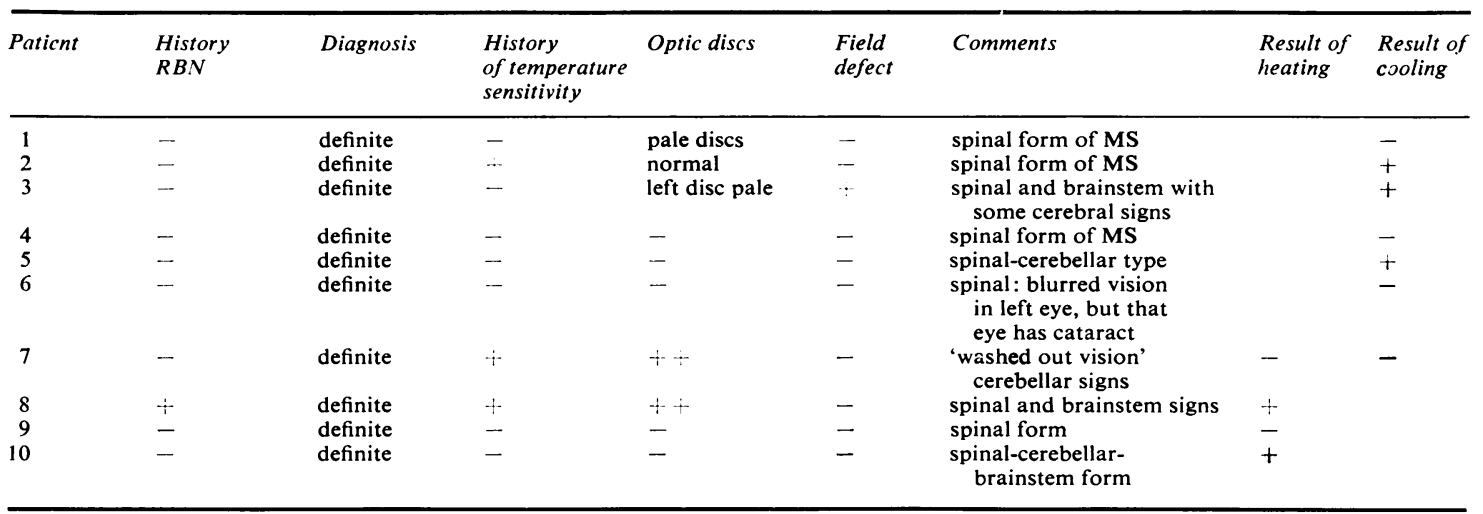

$\mathrm{RBN}=$ retrobulbar neuritis; $\quad \mathrm{MS}=$ multiple sclerosis

\section{Results}

The baseline delay for all 10 patients was outside control limits $(\mathrm{P}<0.05)$ in accord with previous findings (Milner et al., 1974; Regan et al., 1976a, b).

Cooling had no noticeable effect on EP delay (less than $10 \%$ change) in the eight control subjects who were cooled. The control data in Fig. 1A were recorded by means of the single frequency method, and the simultaneous stimulation method was used to obtain the data in Fig. 1B (see Methods).

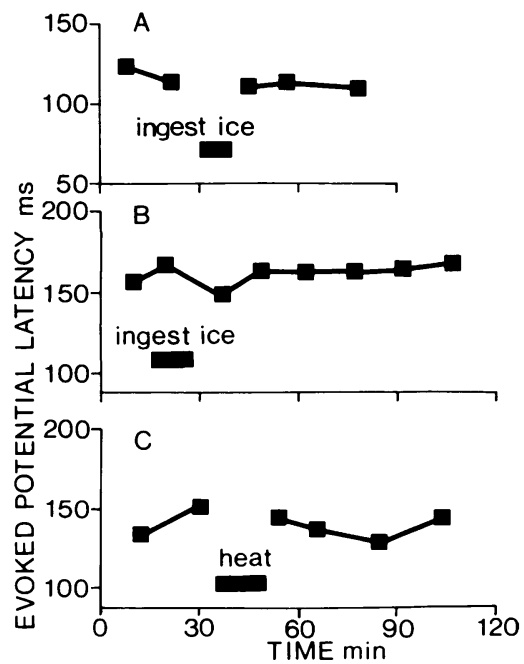

Fig. 1 Neither heating nor cooling produced any systematic changes of evoked potential latency in control subjects. Plot B was recorded using a fast recording technique.
Seven multiple sclerosis patients were cooled. All were diagnosed as having definite multiple sclerosis. Two of these patients showed a clear, temporary reduction of EP delay after ingestion of ice (Fig. 2A, B). One patient's EP delay showed a temporary 'rebound' to a value greater than his baseline (Fig. 2B). Temporary rebounds have been observed previously with visual acuity as a measure (Michael and Davis, 1973). A third patient showed smaller, but still clear temporary reductions in EP latency. The data on the remain-

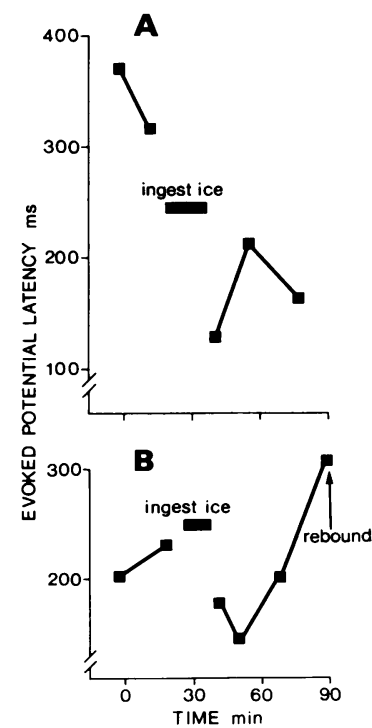

Fig. 2 Cooling reduced evoked potential delay in some multiple sclerosis patients. Other patients did not show this effect. Plot B shows a temporary 'rebound'. A=patient $3 ; B=$ patient 2. 
ing four patients could not be distinguished from control measurements.

Heating had no noticeable effect on EP delay (less than $10 \%$ change) for the four control subjects who were heated. The control data in Fig. 1C were obtained by means of the single stimulation technique (see Methods).

Four multiple sclerosis patients were heated. Two patients ( 8 and 10$)$ showed an increase of latency (Fig. 3A, B). Patient 8 (Fig. 3A) reported 'very hot, vision darkened' at $a$, 'see better' at $b$, and 'back on normal temperature' at $c$. The remaining two patients' data could not be distinguished from control measurements (for example, Fig. 3C).

These EP findings contrast with the results of previous exploratory experiments on the effect of temperature changes upon the delay of visual perception (Regan, Milner, and Heron, unpublished observations). Patients and control subjects wore underclothing which incorporated coils of plastic tubing through which cold water was circulated. The difference in perceptual delays between the left and right eyes was measured as described previously, using a light stimulus of $0.3^{\circ}$ subtense (Regan, 1972b; Heron et al., 1974; Regan et al., 1976b). Measurements were carried out: (a) before cooling; (b) during cooling; (c) one hour after end of cooling. Delays for three control subjects were (in ms): (a) 11, (b) 15 , (c) 8 ; (a) 14 ,

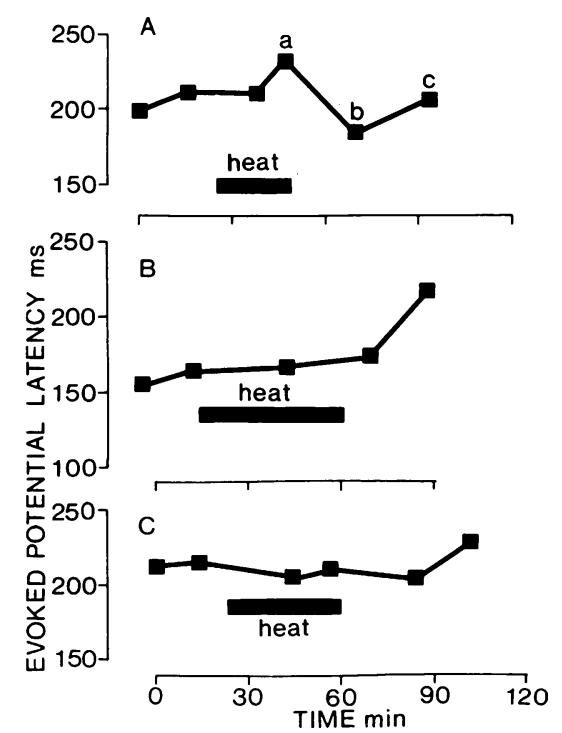

Fig. 3 Heating increased evoked potential delay in some multiple sclerosis patients $(A, B)$, but had no effect in others $(C) . A=$ patient $8 ; B=$ patient 10 ; $C=$ patient 9 . (b) 25 , (c) 4 ; (a) 11 , (b) 11 , (c) 2 . In contrast, cooling caused a clear change of perceptual delay in all seven patients with unilateral retrobulbar neuritis who were tested. Results were as follows: patient 1: (a) 132 , (b) 24 , (c) 14 ; patient 2: (a) 35 , (b) 6, (c) 0 ; patient 3: (a) 41, (b) 36, (c) 11; patient 4: (a) 194, (b) 138, (c) 105; patient 5: (a) 23, (b) -7 , (c) 2; patient 6: (a) 94, (b) 15, (c) 18 . Clearly, delays did not return to precooling values within one hour; but measurements continued for four hours after the end of cooling in one patient (6) showed that a steady value was reached after two hours. A seventh patient was both warmed (twice) and cooled (twice). Baseline values for delay, measured over a two-day period were 126 , $156,165,157,216$, and 127 ms (mean 158). Delays during the two coolings were $105 \mathrm{~ms}$ and $90 \mathrm{~ms}$ and during the two heatings $195 \mathrm{~ms}$ and $180 \mathrm{~ms}$.

One patient who showed no EP effect reported that vision in her left (affected) eye deteriorated noticeably compared with her right eye during the test. We, therefore, assessed her visual acuity thoroughly before, during, and after heating by using a sine wave grating stimulus in the way described elsewhere (Murray et al., 1977; Regan et al., 1977). The sine wave grating test enables visual sensitivity to be assessed for coarse, medium, and fine detail, rather than only for fine detail as in conventional tests such as the Snellen test. The results, shown in Fig 4, confirmed and extended the patient's informal report. Figure 4 plots visual sensitivity (that is, contrast sensitivity) versus spatial frequency (that is, the number of bright bars per degree of visual angle for the grating stimulus). The left eye had previously experienced an attack of retrobulbar neuritis. Preheating data for this eye are marked 1 in Fig. 4A. Plot 2 was recorded during heating and shows a depression of visual contrast sensitivity for all spatial frequencies. Plots 1 and 2 also show that the highest (cutoff) spatial frequency that could be seen with the left eye was also depressed, and this is equivalent to saying that the Snellen acuity would have been depressed. A general depression of visual contrast sensitivity has been reported previously in multiple sclerosis, though as a lasting rather than temporary condition (Regan et al., 1977). Plot 3 was recorded $30 \mathrm{~min}$ after heating ended and shows a 'rebound' to a visual sensitivity higher than before heating. Plot 4 shows that 60 minutes after ending heating the curve was returning to baseline.

Corresponding plots for the right ('unaffected') eye are shown in Fig. 4B. Heating had little effect on the cutoff spatial frequency (equivalent to Snellen acuity). Unexpectedly, it seems that when 

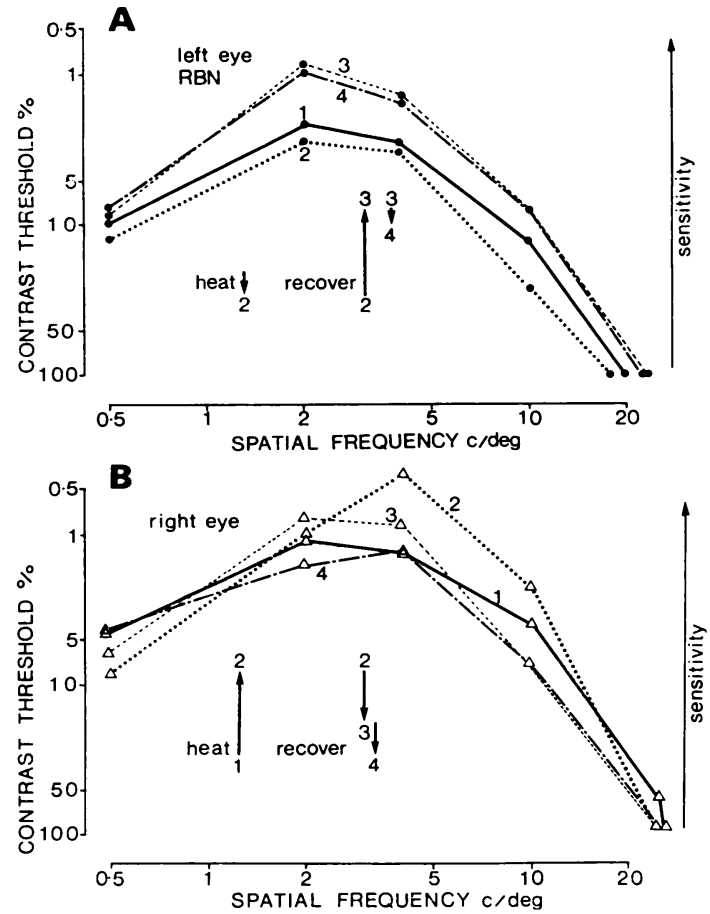

Fig. 4 Effect of heating on visual acuity and spatial contrast sensitivity in patient 7 . The left eye had previously experienced an attack of retrobulbar neuritis. The preheating curve (1) was shifted bodily downwards during heating (2), showing that visual sensitivity was degraded for all spatial frequencies. $V$ isual sensitivity showed an 'overshoot' 30 min after heating was ended (3), but was returning to baseline 60 min after heating was ended (4). Visual acuity (the cutoff spatial frequency) behaved similarly. Visual acuity in the right ('unaffected') eye showed little change. Surprisingly, there was some suggestion that the right eye's sensitivity to medium spatial frequencies increased at about the same time (2) as the left eye's decreased.

the left eye's sensitivity was depressed by heating, the right eye's sensitivity to middle range spatial frequencies was enhanced, finally returning to near baseline levels 60 minutes after the end of heating. Depressing the contrast sensitivity of control subjects by adapting to some value of spatial frequency has been reported not to enhance sensitivity at neighbouring spatial frequencies (Barlow et al., 1976), although enhancement at remote spatial frequencies has been reported (DeValois, 1977).

We measured the delay of visual perception in this patient by a method described previously (Heron et al., 1974; Regan et al., 1976a, b). Figure 5 plots the difference between the perceptual delays

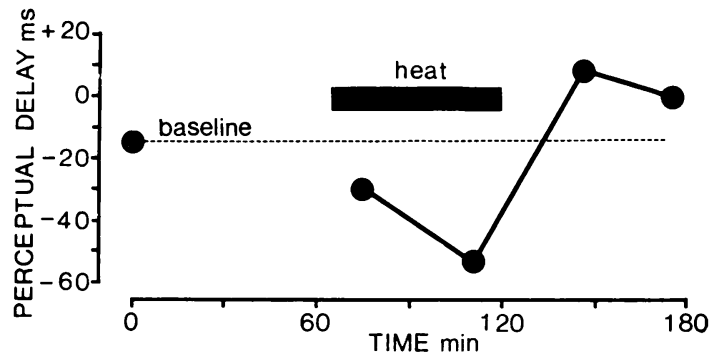

Fig. 5 Effect of heating on the delay of visual perception in patient 7 . Perceptual delay is the difference between left and right fovea: negative values mean the left was slower than the right. Heating increased the delay of the left ('affected') eye compared with the right eye.

for the left and right foveae. Heating increased the delay of the left eye relative to the right eye, and after the cessation of heating there was a 'rebound'. Thus, perceptual delay, but not EP delay, was affected by heating in this patient.

We then measured double-flash resolution in this patient during and after heating. Figure 6 shows the surprising results. Baseline values of double-flash threshold were outside normal limits for both eyes $(\mathrm{P}<0.01)$, but heating improved double-flash resolution. This behaviour is the opposite to our previous observations: double-flash resolution was markedly degraded by heating in all patients previously tested (Galvin et al., 1976b). Note that temperature changes had no effect in control subjects.

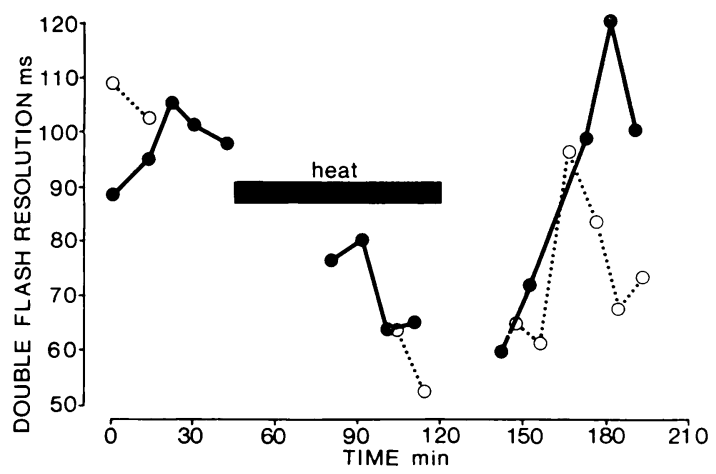

Fig. 6 Anomalous effect of heating on double-flash perception in patient 7 . Ordinates plot the time interval between the onset of two brief flashes for which they are just seen as double rather than single. $-=$ left ('affected') eye, .... = right eye.

Heating improved double-flash resolution for both eyes. All multiple sclerosis patients previously tested showed the opposite effect, and all control subjects showed no effect. 


\section{Discussion}

The visual evoked potential is not a single entity. Among the many different types of visual EP are responses to pattern reversal, responses to flashing a spatially unpatterned light, responses to flickering a spatially unpatterned light at frequencies between 13 and $25 \mathrm{~Hz}$ ('medium frequency' EPs), and 'high frequency' flicker EPs whose frequencies lie between about 40 and 60 $\mathrm{Hz}$ (Regan, 1972a, 1975). There is evidence that of these various types of visual EP several are not only generated in different (though overlapping) areas of cortex, but also reflect the processing of different visual signals in parallel channels at both peripheral and central levels (Regan, 1972a, 1973, 1975).

All these types of EP have been recorded in multiple sclerosis patients with the aim of finding a diagnostically useful indicator of visual pathology. Evoked potentials to pattern reversal of large (50 min arc) checks are delayed in multiple sclerosis (Halliday et al., 1972, 1973; Arden, 1973; Milner et al., 1974; Assleman et al., 1975; McDonald and Halliday, 1977). Evoked potentials to small checks are less reliably delayed (Milner et al., 1974). Evoked potentials to spatially unpatterned flash also seem to be a less reliable indicator of multiple sclerosis than large check EPs (Richey et al., 1971; Halliday et al., 1972), although this view has been challenged (Feinsod and Hoyt, 1975). Medium frequency flicker EPs are reliably delayed in multiple sclerosis (Milner et al., 1974; Regan et al., 1976a). (Figure 3 in Regan (1977b) provides evidence that flicker EPs resemble EPs to large checks or low spatial frequencies, and differ from EPs to small checks or high spatial frequencies.) Three important features of the EP test in multiple sclerosis patients are that some specific types of visual EP show a high incidence of abnormality in multiple sclerosis even when there are no corresponding abnormal clinical signs, that the EP test can demonstrate lasting visual damage even after clinical recovery of vision and that it is an objective test (McDonald and Halliday, 1977; Regan, 1977a). Note, however, that although EPs are not delayed by retinovascular lesions (Milner et al., 1974), multiple sclerosis is not the only cause of delay (Cappin and Nissim, 1975; Halliday et al., 1976; McDonald and Halliday, 1977)

All 10 patients in our present study had delayed EPs. Seven patients were cooled and four heated, but EP latency changed in only five out of 11 experiments: in one case (patient 7) latency was measurably constant to a precision of $6 \%$, though visual changes were noticeable to the patient.
On the other hand, in previous preliminary research, perceptual delay was reduced by cooling in eight out of eight experiments on seven patients, and increased by heating in two out of two experiments. Thus, the effect of body temperature on EP delay seems less consistent than the effects of temperature on perceptual delay.

Double-flash campimetry is a psychological (perceptual) test that can not only detect subclinical visual pathology in multiple sclerosis (in nine out of 11 spinal patients [Galvin et al., 1977]); it can also demonstrate lasting visual damage after acute retrobulbar neuritis (13 out of 14 patients), even when visual acuity has recovered clinically (Galvin et al., 1976a), and can detect the progression of the disease (in 12 cases of advanced multiple sclerosis [Galvin et al., 1977]). In a previous study on four multiple sclerosis patients three were heated and three cooled using similar procedures to ours (Galvin et al., 1976b). Although only one of the 10 patients had previously noticed any visual effects produced by temperature changes, clear alterations in double-flash resolution were produced in all six temperature experiments. Thus EP delay seems to be less sensitive to temperature changes than is double-flash perception. However, although our paradoxical findings with patient 7 may be unusual, they should be kept in mind when using the double-flash test.

Questions could be raised as to the comparability of the results of the various tests. It is, of course, possible that we would have found EP delay to have been affected by temperature in all patients had we heated and cooled the patients more strongly. Our point here, however, is to discuss whether the EP test is less sensitive to temperature changes than are the other tests (and, therefore, presumably less sensitive to changes in demyelination). Since we used the same methods as in the Galvin et al. (1976b) double-flash study it seems unlikely that the heating and cooling procedures were less effective in the present EP study. Although we used a different patient group, their clinical descriptions were not dissimilar. In comparing the results of the EP study with the perceptual delay data reported here it should be kept in mind that heating and cooling procedures were different. On the other hand it seems unlikely that heating was less effective in our EP experiments, as all patients were heated close to their limits of toleration. At any rate, for patient 7 in the present study we used similar heating procedures in all tests.

Differences between the effect of temperature on the EP test and the perceptual tests seem unlikely to be due to the fact that the EP test 
samples a much larger retinal area than either the double-flash or delay tests $\left(48^{\circ}\right.$ compared with $0.3^{\circ}$ diameter). Nevertheless, because of the smal size of the test field the perceptual tests can in principle provide more information than EP tests about the progress of demyelination. By means of visual field plots, double-flash campimetry, and delay campimetry can indicate not only changes in the sizes of 'islands' of demyelination, but also changes in the local severity of the defect (Galvin et al., 1976a, b, 1977).

Although the rule seems to be that (if temperature has any effect at all) heating worsens and cooling improves the signs and symptoms of multiple sclerosis, there are a few reports that signs and symptoms may worsen on exposure to cold (Simons, 1937; Watson, 1959; Geller, 1974). Although, as Geller (1974) pointed out, it is possible that in some of these cases shivering might have caused body temperature to rise on exposure to cold, it nevertheless seems possible that a genuine paradoxical response to temperature changes can occur. A case in point is that heating improved double-flash perception for patient 7 , contrary to experience with other patients. This paradoxical response to heating was peculiar to double-flash, since heating reduced visual acuity and increased perceptual delay. Galvin et al.'s (1976b) finding that the double-flash perception of multiple sclerosis patients was degraded by heating can be understood if heating brings axons closer to their blocking temperatures, and thus reduces the maximum firing frequency (Regan, 1972b; Regan et al., 1976b): an alternative explanation is that adjacent axons have different delays, so that visual signals from different parts of the stimulated retinal area are dispersed in time (McDonald, 1974). Neither explanation can account for the improvement in double-flash perception on heating shown in Fig. 6. Perhaps this anomalous improvement in double-flash perception might be explained if the following unusual situation obtained in this patient. At normal body temperature most fibres were far from their blocking temperature, but conduction was close to failing in some scattered fibres. Conduction along those latter fibres would increase the net temporal dispersion of visual signals so that their selective blockage might improve double-flash resolution. Heating would have little effect on conduction speed for the majority of axons (thus explaining the EP data), except in a few localised regions of more severe demyelination (thus explaining the perceptual delay data). Interestingly, this patient volunteered the report that the perceptual delay test was easier to do during heating than either before or after: presumably simultaneity was more clearly defined.

In this report we explore whether recording medium frequency flicker EPs might provide an objective means of monitoring the progress of demyelination and possibly also of monitoring experimental therapy. We find that, although this EP test provides a reliable indication of visual pathology, EP delay seems to be less affected by changes in body temperature than is either doubleflash perception or perceptual delay. We conclude that the medium frequency flicker EP test seems to be a less sensitive and possibly less reliable means of monitoring the progress of demyelination in multiple sclerosis patients than is doubleflash campimetry or perceptual delay campimetry. However, the objective nature of the EP test would be an advantage in some situations.

We are very grateful to the patients and control subjects who generously assisted in this study. We thank Dr D. B. King for allowing us to examine some of his patients and for supplying diagnostic assessment of them. We are grateful to Annette McCaul for assistance in testing patients. We thank Nancy Beattie for assistance in preparing this manuscript. This work was supported by the Multiple Sclerosis Society of Canada, the Multiple Sclerosis Society of Great Britain and Northern Ireland, and the Medical Research Council (UK). RS was supported as a Summer Student by the Department of Medicine, Camp Hill Hospital and by the Canadian Medical Research Council. Wilkinson Sword Research developed and supplied the double-flash and delay campimeter.

\section{References}

Arden, G. B. (1973). The visual evoked response in ophthalmology, Proceedings of the Royal Society of Medicine, 66, 1037-1044.

Assleman, P., Chadwick, D. W., and Marsden, C. D. (1975). Visual evoked responses in the diagnosis and management of patients suspected of multiple sclerosis. Brain, 98, 261-282.

Barlow, H. B., MacLeod, D. I. A., and van Meeteren, A. (1976). Adaptation to gratings: no compensatory advantages found. Vision Research, 16, 1043-1046.

Boynton, B. L., Garramone, P. M., and Buca, J. T. (1959). Observations on the effect of cool baths for patients with multiple sclerosis. Physical Therapy Review, 39, 297-299.

Cappin, J. M., and Nissim, S. (1975). Visual evoked responses in the assessment of field defects in glaucoma. Archives of Ophthalmology, 93, 9-18.

Davis, F. A. (1966). Hot-bath test in the diagnosis of multiple sclerosis. Journal of the Mount Sinai Hospital, 33, 280-282. 
Davis, F. A. (1970). Axonal conduction studies based on some considerations of temperature effects in multiple sclerosis. Electroencephalography and Clinical Neurophysiology, 28, 281-286.

Davis, F. A., Michael, J. A., and Tomaszewski, J. S. (1973). Fluctuations of motor function in multiple sclerosis related to circadian temperature variations. Diseases of the Nervous System, 34, 33-36.

DeValois, K. K. (1977). Spatial frequency channels revisited: evidence for mutual inhibition. University of Houston Symposium. Abstracts.

Feinsod, M., and Hoyt, W. F. (1975). Subclinical optic neuropathy in multiple sclerosis. Journal of Neurology, Neurosurgery, and Psychiatry, 38, 1109 1114.

Galvin, R. J., Regan, D., and Heron, J. R. (1976a). Impaired temporal resolution of vision after acute retrobulbar neuritis. Brain, 99, 255-268.

Galvin, R. J., Regan, D., and Heron, J. R. (1976b). A possible means of monitoring the progress of demyelination in multiple sclerosis: effect of body temperature on visual perception of double light flashes. Journal of Neurology, Neurosurgery, and Psychiatry, 39, 861-865.

Galvin, R. J., Heron, J. R., and Regan, D. (1977). Subclinical optic neuropathy in multiple sclerosis. Archives of Neurology (Chicago). In press.

Geller, M. (1974). Appearance of signs and symptoms of multiple sclerosis in response to cold. Journal of the Mount Sinai Hospital, 41, 127-130.

Halliday, A. M., and McDonald, W. I. (1977). Pathophysiology of demyelinating disease. British Medical Bulletin, 33(1), 21-27.

Halliday, A. M., McDonald, W. I., and Mushin, J. (1972). Delayed pattern-evoked responses in optic neuritis. Lancet, 1, 982-985.

Halliday, A. M., McDonald, W. I., and Mushin, J. (1973). Delayed pattern-evoked responses in optic neuritis in relation to visual acuity. Transactions of the Ophthalmological Society of the United Kingdom, 93, 315-324.

Halliday, A. M., Halliday, E., Kriss, A., McDonald, W. I., and Mushin, J. (1976). The pattern evoked potential in compression of the anterior visual pathways. Brain, 99, 357-374.

Heron, J. R., Regan, D., and Milner, B. A. (1974). Delay in visual perception in unilateral optic atrophy after retrobulbar neuritis. Brain, 97, 69-78.

Kurtzke, J. F. (1965). On the evaluation of disability in multiple sclerosis. Neurology (Minneapolis), 15, 654-693.

McDonald, W. I. (1974). Pathophysiology in multiple sclerosis. Brain, 97, 179-196.

McDonald, W. I., and Halliday, A. M. (1977). Diagnosis and classification of multiple sclerosis. British Medical Bulletin, 33(1), 4-19.

Michael, J. A., and Davis, F. A. (1973). Effects of induced hyperthermia in multiple sclerosis: differences in visual acuity during heating and recovery phases. Acta Neurologica Scandinavica, 49, 141-151.

Milner, B. A., Regan, D., and Heron, J. R. (1974). Differential diagnosis of multiple sclerosis by visual evoked potential recording. Brain, 97, 755-772.

Murray, T. J., Regan, D., and Silver, R. (1977). Hidden visual loss in multiple sclerosis. Neurology (Minneapolis), 27, 345.

Namerow, N. S. (1968). Circadian temperature rhythm and vision in multiple sclerosis. Neurology (Minneapolis), 18, 417-422.

Namerow, N. S. (1971). Temperature effect on critical flicker fusion in multiple sclerosis. Archives of Neurology (Chicago), 25, 269-275.

Raminsky, M. (1973). The effects of temperature on conduction in demyelinated nerve fibers. Archives of Neurology (Chicago), 28, 287-292.

Regan, D. (1966). Some characteristics of average steady-state and transient responses evoked by modulated light. Electroencephalography and Clinical Neurophysiology, 20, 238-248.

Regan, D. (1972a). Evoked potentials in psychology, sensory physiology and clinical medicine. Chapman and Hall: London, John Wiley: New York.

Regan, D. (1972b). UK Patent Application No. 4865/ 72. US Patent No. 3, 837, 734. W. German No. 2, 304, 808. Wilkinson Sword Ltd.

Regan, D. (1973). Parallel and sequential processing of visual information in man: investigation by evoked potential recording. In Photophysiology, Vol. 8, pp. 185-208. Academic Press: New York.

Regan, D. (1975). Recent advances in electrical recording from the brain (Review). Nature, 253, 401407.

Regan, D. (1976). Latencies of evoked potentials to flicker and to pattern speedily estimated by simultaneous stimulation method. Electroencephalography and Clinical Neurophysiology, 40, 654-660.

Regan, D. (1977a). Visual tests in multiple sclerosis. Proceedings of the San Diego Biomedical Symposium, 16, 87-95.

Regan, D. (1977b). Assessment of visual acuity by evoked potential recording: ambiguity caused by temporal dependence of spatial frequency selectivity. Vision Research. In press.

Regan, D., Milner, B. A., and Heron, J. R. (1976a). Delayed visual perception and delayed visual evoked potentials in the spinal form of multiple sclerosis and in retrobulbar neuritis. Brain, 99, 43-66.

Regan, D., Silver, R., and Murray, T. J. (1977). Visual acuity and contrast sensitivity in multiple sclerosis: hidden visual loss. Brain, 100, 563-579.

Regan, D., Varney, P., Purdy, J., and Kraty, N. (1976b). Visual field analyser: assessment of delay and temporal resolution of vision. Medical and Biological Engineering, 14, 8-14.

Richey, E. T., Kooi, K. A., and Tourtellotte, W. W. (1971). Visual evoked responses in multiple sclerosis. Journal of Neurology, Neurosurgery, and Psychiatry, 34, 275-280.

Rose, A. S., Ellison, G. W., Myers, L. W., and Tourtellotte, W. W. (1976). Criteria for the clinical diagnosis of multiple sclerosis. Neurology (Minneapolis), 26, 20-22.

Schauf, C. L., and Davis, F. A. (1974). Impulse conduction in multiple sclerosis: a theoretical basis for 
modification by temperature and pharmacological agents. Journal of Neurology, Neurosurgery, and Psychiatry, 37, 152-161.

Schumacher, G. A., Beebe, G., and Kilber, R. F. (1968). Problems of experimental trials of therapy in multiple sclerosis: Report of the panel on evaluation of experimental trials in multiple sclerosis. Annals of the New York Academy of Sciences, 122, 522-568.

Simons, D. J. (1937). A note on the effects of heat and cold upon certain symptoms of multiple sclerosis. Bulletin of the Neurological Institute of New York, 6, 385-386.
Symington, G. R., MacKay, I. R., Currie, I. R., and Currie, T. T. (1977). Improvement in multiple sclerosis during prolonged induced hypothermia. Neurology (Minneapolis), 27, 302-303.

Uhthoff, W. (1890). Untersuchungen uber die bei der multiplen herdsklerose vorkommenden augenstorungen. Archiv für Psychiatrie und Nervenkrankheiten, 21, 55-116, 303-410.

Watson, C. W. (1959). Effect of lowering body temperature on the symptoms and signs of multiple sclerosis. New England Journal of Medicine, 261, 1252-1259. 